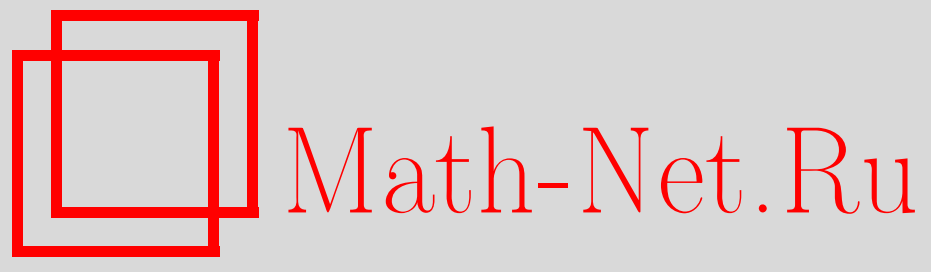

М. И. Фрейдлин, Гомогенизация диффузионных процессов в трубчатых областях, УМН, 2007, том 62, выпуск 3, 219-220

DOI: https://doi.org/10.4213/rm7148

Использование Общероссийского математического портала Math-Net.Ru подразумевает, что вы прочитали и согласны с пользовательским соглашением http://www . mathnet.ru/rus/agreement

Параметры загрузки:

IP: 34.239 .49 .27

26 апреля 2023 г., 15:03:06

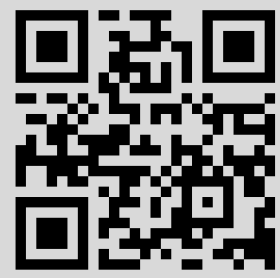




\section{Гомогенизация диффузионных процессов в трубчатых областях}

\section{М. И. Фрейдлин}

Рассмотрим область $D^{\varepsilon} \subset \mathbb{R}^{3}$, представляющую собой трубку вокруг оси $x$, с переменным сечением $D^{\varepsilon}=\left\{(x, y): x \in \mathbb{R}^{1}, \varepsilon^{-1} y \in D_{x}\right\}$. Здесь $0<\varepsilon \ll 1$, сечение $D_{x}$ есть ограниченная область в $\mathbb{R}^{2}$, содержащая начало координат. Предполагается, что область $D^{1}=\left.D^{\varepsilon}\right|_{\varepsilon=1}$ имеет достаточно гладкую границу $\partial D^{1}$ и нормаль к $\partial D^{1}$ никогда не параллельна оси $x$.

Обозначим $Z_{t}^{\varepsilon}=\left(X_{t}^{\varepsilon}, Y_{t}^{\varepsilon}\right)$ винеровский процесс в $D^{\varepsilon}$ с нормальным отражением на границе. Его траектории можно описать уравнениями

$$
d X_{t}^{\varepsilon}=d W_{t}^{1}+\gamma_{1}^{\varepsilon}\left(X_{t}^{\varepsilon}, Y_{t}^{\varepsilon}\right) d l_{t}^{\varepsilon}, \quad d Y_{t}^{\varepsilon}=d W_{t}^{2}+\gamma_{2}^{\varepsilon}\left(X_{t}^{\varepsilon}, Y_{t}^{\varepsilon}\right) d l_{t}^{\varepsilon},
$$

где $W_{t}^{1}$ и $W_{t}^{2}$ - независимые одномерный и двумерный винеровские процессы соответственно; $l_{t}^{\varepsilon}$ - локальное время на $\partial D^{\varepsilon} ; \gamma_{1}^{\varepsilon}(x, y)$ и $\gamma_{2}^{\varepsilon}(x, y)$ - проекции единичного вектора внутренней нормали на ось $x$ и на плоскость $\left(y_{1}, y_{2}\right)$ соответственно.

В этой работе мы изучаем поведение компоненты $X_{t}^{\varepsilon}$ процесса $Z_{t}^{\varepsilon}$ при $\varepsilon \ll 1$ и $t \gg 1$, когда область $D^{\varepsilon}$ в том или ином смысле однородна по $x$. Можно ожидать, что в этом случае $x$-компонента процесса $Z_{t}^{\varepsilon}$ хорошо аппроксимируется процессом с постоянным коэффициентом диффузии и нулевым сносом. Мы вычисляем этот коэффициент явно. Мы рассматриваем также уравнение реакции-диффузии в области $D^{\varepsilon}$.

Обозначим $S(x)$ площадь сечения $D_{x}$.

Теорема 1. Предположим, что $S(x), x \in \mathbb{R}^{1}$, есть эргодический стачионарный процесс. Тогда случайный процесс $\widetilde{X}_{t}^{\varepsilon, \delta}=\delta X_{t / \delta^{2}}^{\varepsilon}$ сходится слабо в пространстве непрерывных функиий $C[0, T]$ на отрезке $[0, T], T>0, \kappa$ процессу $\sigma W_{t}$, где $W_{t}-$ одномерный винеровский процесс $u \sigma=\left(\mathrm{E} S(x) \mathrm{E} \frac{1}{S(x)}\right)^{-1 / 2}$, если сначала $\varepsilon \rightarrow 0$, затем $\delta \rightarrow 0$ (Е - знак математического ожидания).

Если функиия $S(x)$ периодична, $S(x) \equiv S(x+1)$, то $\widetilde{X}_{t}^{\varepsilon, \delta}$ сходится слабо в $C[0, T]$, когда сначала $\varepsilon \rightarrow 0$, а затем $\delta \rightarrow 0, \kappa$ прочессу $\widetilde{\sigma} W_{t}$, где

$$
\widetilde{\sigma}=\left(\int_{0}^{1} S(x) d x \int_{0}^{1} \frac{d x}{S(x)}\right)^{-1 / 2} .
$$

Доказательство этой теоремы состоит из двух частей. Сначала мы показываем, что при $\varepsilon \downarrow 0$ процессы $X_{t}^{\varepsilon}$ на любом конечном отрезке $[0, T]$ слабо сходятся к одномерному диффузионному процессу $X_{t}$, соответствующему оператору $L=\frac{1}{2} \frac{d^{2}}{d x^{2}}+\frac{1}{2} \frac{S^{\prime}(x)}{S(x)} \frac{d}{d x}$. Этот результат (ср. с [1; гл. 7]) представляет собой вариант принципа усреднения. Чтобы доказать сходимость $X_{t}^{\varepsilon}$ к $X_{t}$, рассмотрим вспомогательную функцию $v(x, y)$, $x \in \mathbb{R}^{1}, y \in \mathbb{R}^{2}$, которая есть решение следующей задачи в области $D_{x} \subset \mathbb{R}^{2}, x-$ параметр:

$$
\frac{1}{2} \Delta_{y} v(x, y)=A(x), \quad y \in D_{x},\left.\quad \frac{\partial v}{\partial n}\right|_{\partial D_{x}}=\frac{\gamma_{1}^{1}(x, y)}{\gamma_{2}^{1}(x, y)},
$$

где $n=n(x, y)$ - вектор внутренней нормали к $\partial D_{x}$ в плоскости сечения $D_{x}, \gamma_{i}^{1}(x, y)$, $i=1,2,-$ значение $\gamma_{i}^{\varepsilon}(x, y)$ для $\varepsilon=1$. Для всякого $x \in \mathbb{R}^{1}$ задача (2) разрешима, только если

$$
A(x)=-\frac{1}{S(x)} \oint_{\partial D_{x}} \frac{\gamma_{1}^{1}}{\left|\gamma_{2}^{1}\right|} d l, \quad d l-\text { элемент длины на } \partial D_{x} .
$$

Решение задачи (2) единственно с точностью до аддитивной функции $c=c(x)$. Эту функцию $c(x)$ можно выбрать так, что решение $v(x, y), x \in \mathbb{R}^{1}, y \in D_{x} \subset \mathbb{R}^{2}$, задачи (2) будет иметь непрерывные вторые производные по $x$ и $y$. Применяя формулу 
Ито к функции $v\left(X_{t}^{\varepsilon}, \varepsilon^{-1} Y_{t}^{\varepsilon}\right)$, где $\left(X_{t}^{\varepsilon}, Y_{t}^{\varepsilon}\right)$ - решение задачи (1), и принимая во внимание, что $\lim _{\varepsilon \rightarrow 0} \varepsilon^{-1} \gamma_{1}^{\varepsilon}=\gamma_{1}^{1}$ и $\lim _{\varepsilon \rightarrow 0}\left|\gamma_{2}^{\varepsilon}\right|=1$, нетрудно заключить, что

$$
\max _{0 \leqslant t \leqslant T}\left|X_{t}^{\varepsilon}-\widetilde{X}_{t}\right| \rightarrow 0 \quad \text { при } \quad \varepsilon \rightarrow 0
$$

по вероятности, где $\widetilde{X}_{t}$ - решение уравнения

$$
d \widetilde{X}_{t}=d W_{t}^{1}+\frac{1}{2} A\left(\widetilde{X}_{t}\right), \quad \widetilde{X}_{0}=X_{0}^{\varepsilon}=x .
$$

Так как $\oint_{\partial D_{x}} \gamma_{1}^{1}(x, y) d l=S^{\prime}(x)$, мы заключаем, что процесс $\widetilde{X}_{t}$ управляется оператоpом $L$.

Сходимость процесса $\delta \widetilde{X}_{t / \delta^{2}}$ к $\sigma W_{t}$ есть результат гомогенизации. Здесь можно воспользоваться условиями, обеспечивающими слабую сходимость процессов, приведенными в [2].

Рассмотрим теперь задачу о движении волнового фронта в уравнении реакциидиффузии

$$
\begin{gathered}
\frac{\partial u^{\varepsilon}(t, x, y)}{\partial t}=\frac{1}{2} \Delta u+f(u), \quad t>0, \quad(x, y) \in D^{\varepsilon}, \\
\left.\frac{\partial u^{\varepsilon}}{\partial n^{\varepsilon}}\right|_{\partial D^{\varepsilon}}=0, \quad u^{\varepsilon}(0, x, y)=\chi_{-}(x)= \begin{cases}1, & x \leqslant 0 \\
0, & x>0\end{cases}
\end{gathered}
$$

Предполагается, что $f(u)=c(u) u$ удовлетворяет условиям Колмогорова-Петровского-Пискунова: $c(u)>0$ при $u \in(0,1), c(1)=0, c(0)=c=\max _{u \in[0,1]} c(u), c(u)$ удовлетворяет условию Липшица.

Теорема 2. Предположим, что функиия $S(x)$ периодична с периодом 1. Рассмотрим следуюшую задачу на собственные значения:

$$
\begin{gathered}
\frac{1}{2} \varphi^{\prime \prime}(x)+\frac{1}{2} \frac{S^{\prime}(x)}{S(x)} \varphi^{\prime}(x)-z \varphi^{\prime}(x)+\left(c+\frac{1}{2} \frac{S^{\prime}(x)}{S(x)} z+\frac{z^{2}}{2}\right) \varphi(x)=\lambda(z) \varphi(x), \\
\varphi(x) \equiv \varphi(x+1), \quad z \in \mathbb{R}^{1}-\text { nараметр. }
\end{gathered}
$$

Для каждого $z \in \mathbb{R}^{1}$ эта задача имеет однократное собственное значение $\lambda=\lambda(z)$, соответствующее положительной собственной функиии. Функиия $\lambda(z)$ непрерьвно дифберенцируема и выпукла. Обозначим $H(y)$ ее преобразование Лежандра: $H(y)=$ $\sup _{z \in \mathbb{R}^{1}}(y z-\lambda(z))$. Тогда

$$
\lim _{t \rightarrow \infty} \lim _{\varepsilon \rightarrow 0} u^{\varepsilon}(t, t y)= \begin{cases}0, & \text { если } H(y)>0, \\ 1, & \text { если } H(y)<0 .\end{cases}
$$

Доказательство этой теоремы основано на слабой сходимости $X_{t}^{\varepsilon}$ при $\varepsilon \rightarrow 0$, которая используется в предыдущей теореме, и на результатах $[3 ; \S 7.3]$.

\section{Список литературы}

[1] M. Freidlin, Markov processes and differential equations: asymptotic problems, Lectures Math. ETH Zurich, Basel, Birkhäuser, 1996. [2] M. Freidlin, A. Wentzell, The Dynkin Festschrift, Progr. Probab., 34, ed. M. Freidlin, Boston, MA, Birkhäuser, 1994, 95-109. [3] M. Freidlin, Functional integration and partial differential equations, Ann. of Math. Stud., 109, Princeton Univ. Press., Princeton, 1985.

М. И. Фрейдлин (M. I. Freidlin)

University of Maryland, USA

E-mail: mif@math.umd.edu
Представлено С. П. Новиковым Принято редколлегией 16.03.2007 